\title{
Statistical tools of interstellar turbulence: connecting observations with theory
}

\author{
B. Burkhart \& A. Lazarian \\ Astronomy Department, University of Wisconsin, Madison, 475 N. Charter St., WI 53711, USA \\ email: burkhart@astro.wisc.edul
}

\begin{abstract}
MHD Turbulence is a critical component of the current paradigms of star formation, particle transport, magnetic reconnection and evolution of the ISM, to name just a few. Progress on this difficult subject is made via numerical simulations and observational studies, however in order to connect these two, statistical methods are required. This calls for new statistical tools to be developed in order to study turbulence in the interstellar medium. Here we briefly review some of the recently developed statistics that focus on characterizing gas compressibility and magnetization and their uses to interstellar studies.
\end{abstract}

Keywords. turbulence, methods:statistical numerical, ISM: general

\section{Introduction}

The paradigm of the interstellar medium has undergone major shifts in the past two decades thanks to the combined efforts of high resolution surveys and the exponential increase in computation power allowing for more realistic numerical simulations. The ISM is now known to be highly turbulent and magnetized, which affects ISM structure, formation, and evolution. Magnetohydrodynamic turbulence is essential to many astrophysical phenomena such as star formation, cosmic ray dispersion, magnetic reconnection, and many transport processes. However the study of turbulence is complicated by the fact that no complete theory for turbulence exists.

To this date, turbulence has always been understood in a statistical manner - showing the 'order from chaos.' The classical picture of Kolmogrov 1941 depicts turbulent flows as composed of eddies which transfer energy across a range of scales, typically from the larger injection scale down to what is know as the dissipative range. The eddies are unstable and break into smaller and smaller eddies, transferring their kinetic energy in a manner that conserves it over time. This picture of the 'energy cascade' is highly dependent on the characteristic dimensionless Reynolds number, which describes the ratio of the inertial to viscous forces. By simple dimensional analysis it can be shown that the energy spectrum scales as $k^{-5 / 3}$. More current research shows that turbulence is intermittent and not statistically self-similar, as is assumed in the Kolmogrov picture. To add to the complexity, the ISM is in a plasma state and gas dynamics are governed by the MHD equations, which changes the the scaling relationships via interactions of MHD waves and Alfvénic shearing.

In addition to the Reynolds number, other important parameters of ISM MHD turbulence include the sonic Mach number, which describes the compressibility of the gas as $\mathcal{M}_{s} \equiv \mathbf{v} / C_{s}$, and the Alfvénic Mach number $\mathcal{M}_{A} \equiv \mathbf{v} / v_{A}$, where $v_{A}=|\mathbf{B}| / \sqrt{\rho}$ is the Alfvénic velocity, $\mathbf{B}$ is magnetic field and $\rho$ is density. These parameters are not always easy to characterize observationally, with the Alfvénic Mach number being particularly difficult due to cumbersome observational measurements of vector magnetic field.

Several techniques have been around for decades in order to study ISM turbulence and its properties. Many of these hinge on either density fluctuations, via scintillation in ionized media, Radio position-position-velocity data (PPV) and column density maps 


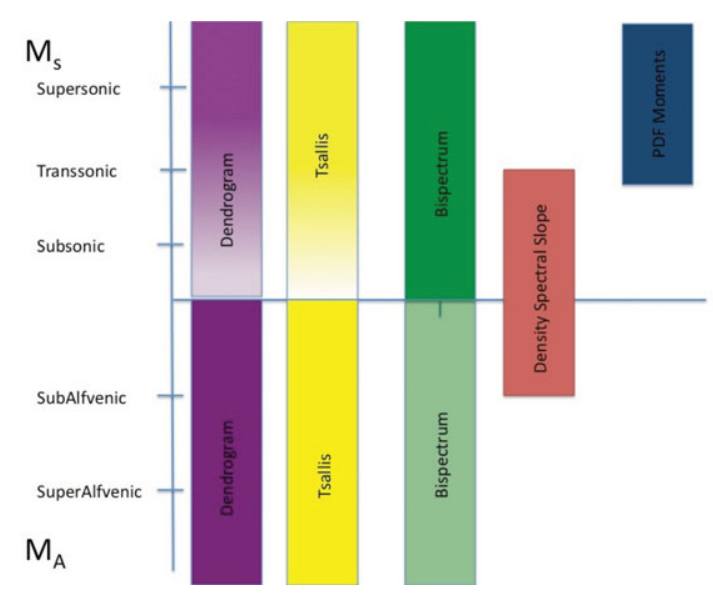

Figure 1. Cartoon showing different statistics studied with their dependencies on the sonic and Alfvénic Mach numbers. The different colors indicate different staitistics. The intensity of the colors indicate the confidence the statistics can provide the parameter on the y-axis in our simulations.

for neutral media. The advantage of spectroscopic data is that it contains information about the turbulent velocity field as well as the density fluctuations, however separation of the density and velocity fields is non trivial (Lazarian 2009). When invoking the phenomena of turbulence many researchers base their analysis around the slope of the log-log spatial power spectrum. While the power spectrum gives information about the energy per wavenumber (or frequency), it only contains the Fourier amplitudes and completely ignores the phases. This alone is motivation for the development of complimentary techniques.

In general, the best strategy for studying a difficult subject like interstellar turbulence is to use a synergetic approach, combining theoretical knowledge, numerical simulations, and observational data via statistical studies. In this way one can obtain the most complete and reliable picture of the physics of turbulence. Here we seek to extend the statistical comparison between numerical and observational turbulence by reviewing statistical tools that can greatly compliment the information provided by the power spectrum. In particular we focus the review on tools that can provide information on $\mathcal{M}_{s}$ and $\mathcal{M}_{A}$. This review will highlight several different tools (see Figure 1) studied in the works of Kowal et al., (2007), Burkhart et al., (2009), (2010), (2010b), Esquivel et al., (2010), Toffelmire et al., (2010), Chepurnov \& Lazarian (2009), Rosolowsky et al., (2008), and Goodman et al., (2009), which represent a mixture of numerical and observational studies. We focus on statistics that have application for observable data (PPV or column density).

\section{Supersonic versus Subsonic Turbulence}

The sonic Mach number describes the ratio of the flow velocity to the sound speed, and thus is a measure of the compressibility of the medium. Turbulence that is supersonic displays very different characteristics then subsonic turbulence in terms of the spectral slope and density/ velocity fluctuations. Because the physical environment of compressible turbulence is very different from incompressible, this parameter is extremely important for many different fields of astrophysics including, but not limited to, star formation and cosmic ray acceleration.

\subsection{Higher Order Moments of Column Density}

Moments of the density distribution can be used to roughly determine the gas compressibility through shock density enhancements. As the ISM media transitions from subsonic to supersonic and becomes increasingly supersonic, shocks create enhanced mean value and variance of the density PDF. In addition, as the shocks become stronger, the PDF is 
skewed and becomes more kurtotic then Gaussian. However, Kowal et al., (2007) showed that this method is not very effective for subsonic cases, as these distributions are roughly Gaussian. For areas where Mach numbers approach and exceed unity, higher order moments of column density PDFs can be used as a measure of compressibility.

\subsection{Spectrum}

The power spectrum has been used for studies of both observational and numerical turbulence for decades. In addition to providing information on the energy cascade, the spectrum can also be used to obtain compressibility in column density maps. As turbulence transitions from subsonic to supersonic, density enhancements due to shocks create small scale structures which shallow out the spectral slope. Additionally, the presence of a strong magnetic field can create Alfvénic shearing, which can steepen the slope via destruction of small scale structures. Subsonic low magnetic field spectral slope values are close to what is predicted for hydrodynamic Kolmogorov turbulence and the addition of a strong magnetic field and shocks cause deviations from the predicted $-11 / 3$. $\dagger$ Burkhart et al., (2010) compared the spectral slope found for the SMC galaxy with simulations of turbulence and found that the SMC's slope of -3.3 matches well with transsonic type turbulence. This Mach number range has been independently confirmed by observational methods to obtain the sonic number which utilize the ratio of the spin the kinetic temperature and also the higher order moments.

\subsection{Bispectrum}

While the power spectrum has been used extensively in ISM studies, higher order spectrum have been more rare. The bispectrum, or Fourier transform of the 3rd order autocorrelation function, has been applied to isothermal ISM turbulence simulations and the SMC only recently (Burkhart et al., 2009, 2010) although it is extensively used in other fields including cosmology and biology.

The bispectrum preserves both the amplitude and phase and provides information on the interaction of wave modes. Completely randomized modes will show a bispectrum of zero, while mode coupling will show non-zero bispectrum. Shocks and high magnetic field have been shown to increase mode coupling in the bispectrum. Due to their ability to shallow out the density energy spectrum, shocks greatly enhance the small scale wavewave coupling. With simulations with the same sonic number, mode correlation is shown to increase with an increase in magnetic field, however this is less clear for column density as it is for $3 \mathrm{D}$ density.

\section{Magnetization of Turbulence}

The Alfvén number is the dimensionless ratio of the flow velocity to the Alfvén speed. As the Alfvén speed depends on the magnetic field, this ratio can provide information on the strength of the magnetic field relative to the velocity and density. The Alfvénic number is critical in several fields including interplanetary studies and star formation. The solar wind is known to be a super-Alfvénic flow while the Alfvénic number in star forming regions is still hotly debated.

\subsection{Tsallis PDFs of PPV and Column Density}

PDFs of increments (of density, magnetic field, velocity etc.) are a classic way to study turbulence since the phenomena is scale dependent. The Tsallis function was formulated in Tsallis (1988) as a means to extend traditional Boltzmann-Gibbs mechanics to fractal and multifractal systems.

$$
R_{q}=a\left[1+(q-1) \frac{\Delta f(x, r)^{2}}{w^{2}}\right]^{-1 /(q-1)}
$$

$\dagger$ For incompressible turbulence, the Kolmogorov power spectrum in three dimensions (3D) is $k^{-11 / 3}$, in $2 \mathrm{D}$ it is $k^{-8 / 3}$, and $1 \mathrm{D} k^{-5 / 3}$ for the same energy spectrum $\mathrm{E}(\mathrm{k})$. 
The Tsallis distribution (Equation 3.1) can be fit to PDFs of increments, that is, $f(x, r)=G(x+r)-G(x)$, where $\mathrm{G}(\mathrm{x})$ is a particular field (for example, turbulent density, velocity or magnetic field). The Tsallis fit parameters ( $\mathrm{q}$, a, and $\mathrm{w}$ in Equation 3.1) describe the width, amplitude, and tails of the PDF. These parameters have been shown to have dependencies on both sonic and Alfvénic Mach number. Tsallis parameters are able to distinguish between sub, tras and supersonic turbulence as well as gauge wether the turbulence is sub-Alfvénic or super-Alfvénic. The parameter that describes the width of the PDF distribution is particularly sensitive.

\subsection{Dendrograms of Position-Position-Velocity (PPV) data}

A Dendrogram (from the Greek dendron tree,- gramma drawing) is a hierarchical tree diagram that has been used extensively in other fields, particular galaxy evolution and biology. It is a graphical representation of a branching diagram, and for our particular purposes with PPV data, quantifies how and where local maxima of emission merge with each other. The dendrogram was first used on ISM data in Rosolowsky et al., 2008 and Goodman et al., 2009 in order to characterize self-gravitating structures in star forming molecular clouds.

Burkhart et al., 2010 used the dendrogram on synthetic PPV cubes and found it to be rather sensitive to magnetic density/velocity enhancements. They looked at the moments of the distribution of local maxima in emission found in the tree diagram. These moments showed clear signs of being dependent on Mach numbers, with the particular strength being the Alfvén number. When high frequency filtering is applied in order to mask small scale enhancements due to shocks, the magnetic enhancements fully dominate the moments of the tree diagram distribution. The dendrogram is also able to distinguish between simulations that show varying degrees of gravitational strength. It is also very encouraging that this statistic is working in PPV space, while other statistics studied utilize the column density maps. This further motivates the synergetic approach of using these statistics.

\section{Conclusions}

The last decade has seen major increases in the knowledge of the ISM and of its turbulent nature thanks to high resolution observations and advanced numerical simulations. This calls for new advances in statistical tools in order to best utilize the wealth of observational data in light of numerical and theoretical predictions. Recently several authors have explored new tools for studying turbulence beyond the power spectrum. While these proceedings do not cover all the useful tools in the literature, we attempt to provide some review on tools that describe the gas compressibility and the Alfvénic Mach number by utilizing density fluctuations created by shocks and magnetic density enhancements.

\section{References}

Burkhart, B., Falceta-Goncalves, D., Kowal, G., \& Lazarian, A., 2009, ApJ, 693, 250

Burkhart, B., Stanimirovic, S., Lazarian, A., \& Kowal, G., 2010, ApJ, 708, 1204

Burkhart, B., Goodman, A., Lazarian, A., \& Rosolowsky, E., 2010, in prep.

Chepurnov, A., Lazarian, A., Gordon, J., \& Stanimirovic., S., 2008, ApJ, 688, 1021

Esquivel, A. \& Lazarian, A., 2010, ApJ, 710, 125

Goodman, A. A., Rosolowsky, E. W., Borkin, M. A., Foster, J. B., Halle, M., Kauffmann, J. \& Pineda, J. E., 2009, Nature letters, 457, 63

Lazarian, A. 2009, Space Science Reviews, 143, 357

Kowal, G., Lazarian, A., \& Beresnyak, A., 2007, ApJ, 658, 423

Toffelmire B., Burkhart, B., \& Lazarian, A., 2010, submitted.

Rosolowsky, E. W., Pineda, J. E., Kauffmann, J., \& Goodman, A. A. 2008, ApJ, 679, 1338 